chlorpromazine from its complex with ribuflavin or flavin monophosphate was also calculated to be $1 \times 10^{-3} M$ from fluorimetry.

From these results, it may be concluded that chlorpromazine forms a complex with the isoalloxazine part of flavins at a concentration of $10^{-3} \mathrm{M}$. Considering the order of the dissociation constants of the complexes calculated from fluorimetry, it is clear that complex formation can be excluded from the mechanism of inhibition of $\mathrm{D}$-amino acid oxidase by chlorpromazine. However, the complex formation of chlorpromazine with flavins could be significant in vivo if higher concentrations of chlorpromazine than $10^{-5} M$ were reached. For example, the fact that the injection of flavin adenine dinucleotide can reverse the effects of chlorpromazine on an electroencephalogram can be partly explained by the formation of a complex of these two compounds in the living body5.

KUNIO YAGI

TAKaYUKI Ozawa

Department of Biochemistry.

Toshrharu Nagatsu

Department of Neuropsychiatry,

School of Medicine,

Nagoya University, Nagoya. May 22

\footnotetext{
Yagi, K., Okuda, J., Ozawa, T., and Okada, K., Science, 124, 273 (1956); Biochim. Biophys. Acta (in the press)

${ }^{2}$ Yagi, K., Okuda, J., Ozawa, T., and Okada, K., Biochem. Z., 328, 492 (1957).

Yagi, K., Nagatsu, T., and Ozawa, T., Nature, 177, 891 (1956)

Szent-Györgyi, A., 'Bioenergetics', 118 (Academic Press, 1957)

agi, K., Ozawa, T., Ando, M., and Nagatsu, T. (unpulished
}

\section{Preparation of a Highly Purified Sample of the Urinary Gonadotrophin}

THE difficulty encountered in the determination of the physical and chemical properties of the urinary gonadotropin is closely related to its small concentration in urine. It is also the reason why so many studies concerning this hormone refer mainly to its biological properties. Nevertheless, it would be of considerable interest to obtain gondadotropin of high purity. That is why we attempted to isolate it from the urine of sterilized women or women at the menopause. We report here our preliminary results.

The urine is adjusted to $p \mathrm{H} 4.5$ and 3 parts of 95 per cent alcohol are added. The precipitate is extracted 3 times with 50 per cent alcohol. The residue is separated by centrifugation and the remaining liquid is concentrated to a 75 per cent alcoholic solution. The precipitate is washed, dried and dis-

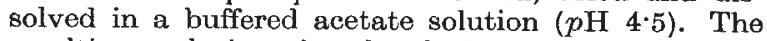
resulting solution is absorbed on kaolin'. The ammoniacal eluate is adjusted to $p H 5 \cdot 1$ then 3 parts of acetone are added. The precipitate which is formed is centrifuged, washed and dried. It is then passed through an ion exchange resin ('Permutit' or 'Dowex'). The product is purified without appreciable loss. The association of two ion-exchange resins brings no jmprovement.
The preparation thus obtained reveals a strong activity in the test on the gain of weight of the uterus of immature mice ${ }^{2}$.

Table 1 shows the increase in purity at the different stages ( 2 experiments):

The glycoproteic nature of the preparation was demonstrated by the determination of the hexoses (13 per cent), hexosamines (11 per cent) and the high percentage of sialic acid ( 8 per cent). Electrophoresis in the liquid phase according to Tiselius ${ }^{3}$ at $p \mathrm{H}=8,6$, $\mu=0 \cdot 1$, revealed the non-homogeneous quality of the product. Finally, the product was tested histologically on the ovaries of immature rats hypophysectomized 2 months before. A distinct follicular maturation was observed together with a protoplasmic hypertrophia of the interstitial cells.

Thus even at this degree of purity the preparation possesses both follicle-stimulatory and luteinizing stimulatory properties.

\section{Roland Bourrillon}

RENÉ GOT

RenÉ Marcy

Laboratoire de Biochimie,

Faculté de Médecine,

45 Rue des Saints-Pères, Paris 6.

April 10.

1 Lorraine, J. A., and Brown, J. B., Acta Endocrinol., 17, 250 (1954)

Klinefelter, H. F., Albright, F., and Griswold, G. C., J. Clin. Endocrinol. Metabol., 3, 529 (1943)

s Tiselius, A,, Trans. Faraday Soc., 33, 524 (1937).

\section{Biosynthesis}

Using methyl and carboxyl labelled acetate, Grob and Butler ${ }^{1,2}$ have shown that both carbon atoms of acetate are extensively incorporated into $\beta$-carotene synthesized by Mucor heimalis. They also found that pantothenic acid and pantetheine stimulate $\beta$-carotene synthesis; on the basis of this fact they have suggested that coenzyme $A$ is involved in carotenogenesis. Studies have therefore been made to investigate the role of coenzyme $A$ in the biogenesis of carotenoids; the present study details the results of the experiments on carotene biosynthesis using extracts prepared from carrots (Daucus carota).

The carrots after removal from the soil were cooled in crushed ice, and in the frozen condition they were cut into small pieces. $10 \mathrm{gm}$. (fresh wt.) were ground with $100 \mathrm{ml}$. phosphate buffer $(0.2 \mathrm{M}, \mathrm{pH}$ $5 \cdot 8$ ) at $0^{\circ} \mathrm{C}$. for about $10 \mathrm{~min}$. and the fine debris removed by centrifugation at $500 \mathrm{~g}$. for 5 min. The supernatant solution of the carrot extract (containing protein concentration of about $6 \mathrm{mgm}$.) were incubated at $28^{\circ} \mathrm{C}$. in $250-\mathrm{ml}$. Erlenmeyer flasks on a rotary shaker for $18 \mathrm{hr}$. with the desired substrates in phosphate buffer $(p \mathrm{H} 5.8,0.2 M$.) Carotenes were extracted in freshly distilled ether. The etheral extract was freed of moisture by treating with anhydrous sodium sulphate. The carotenes were transferred in $5 \mathrm{ml}$. petroleum ether (b.p. $80-100^{\circ}$ C.) and were determined as $\beta$-carotene Table 1

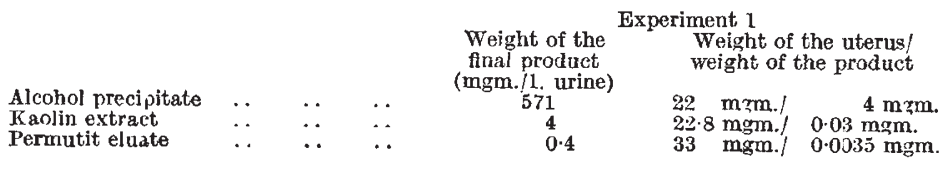

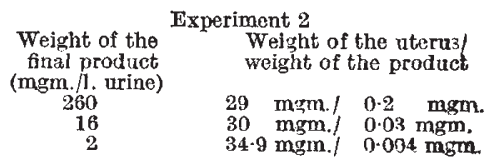

